\title{
Relief Articulation Techniques
}

\author{
Jan Koenderink ${ }^{1,2}$, Andrea van Doorn ${ }^{2}$, Liliana Albertazzi ${ }^{3,4}$ and Johan Wagemans ${ }^{1}$ \\ ${ }^{1}$ Laboratory of Experimental Psychology, University of Leuven (KU Leuven), Belgium \\ ${ }^{2}$ Experimental Psychology, Utrecht University, The Netherlands \\ ${ }^{3}$ Center for Mind/Brain Sciences (CIMeC), University of Trento, Italy \\ ${ }^{4}$ Department of Humanities, University of Trento, Italy
}

\section{Supplementary Figures}

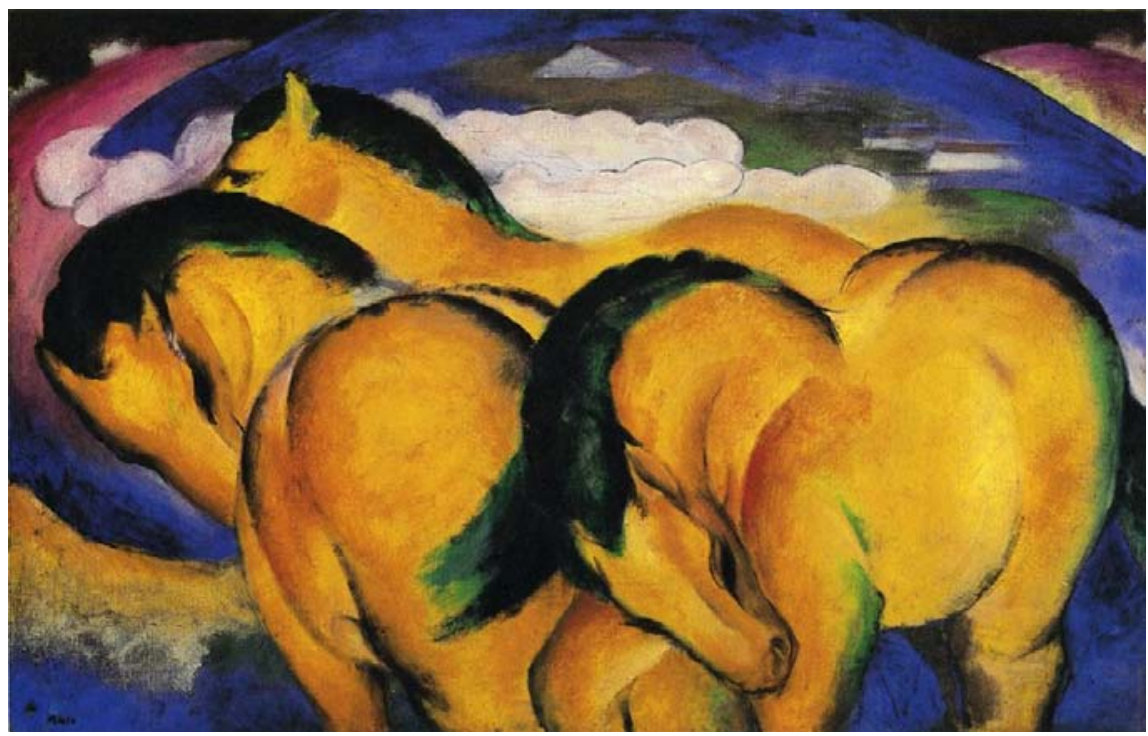

Figure S1. A painting by Franz Marc (1880-1916). The "shading” is largely concentrated in ridges and ruts, that are curve-like, rather than area-like parts. We refer to it as contour modulation, mainly by way of bipolar edges, and some degree of edge darkening. 


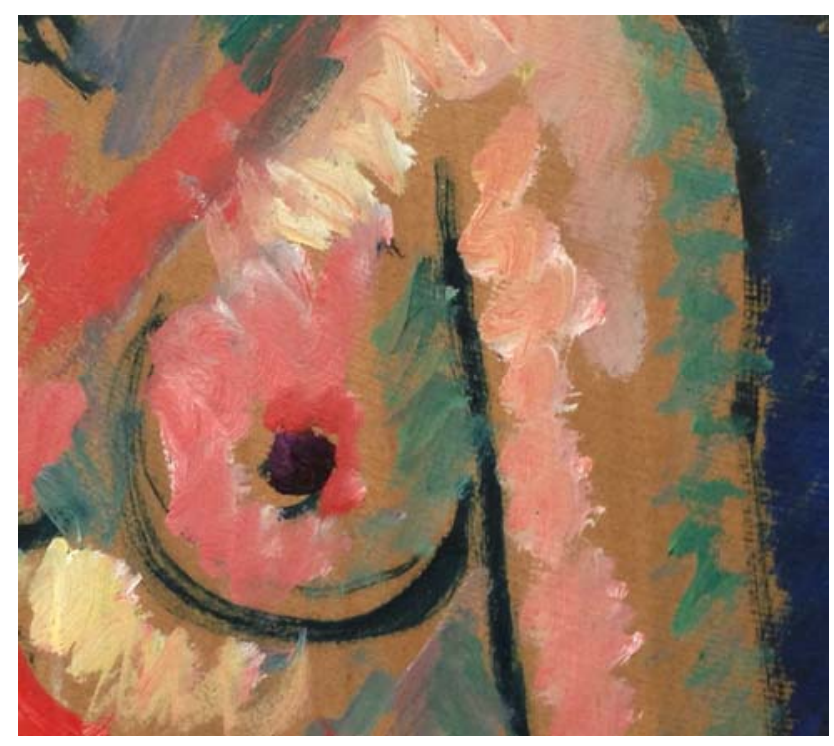

Figure S2. Detail of a nude by Alexej von Jawlenski (1864 or 1865-1941). It shows a sphere (breast) and a cylinder (arm), "shaded" in red-green instead of light-dark. This is chromatic modulation emulating proper shading.
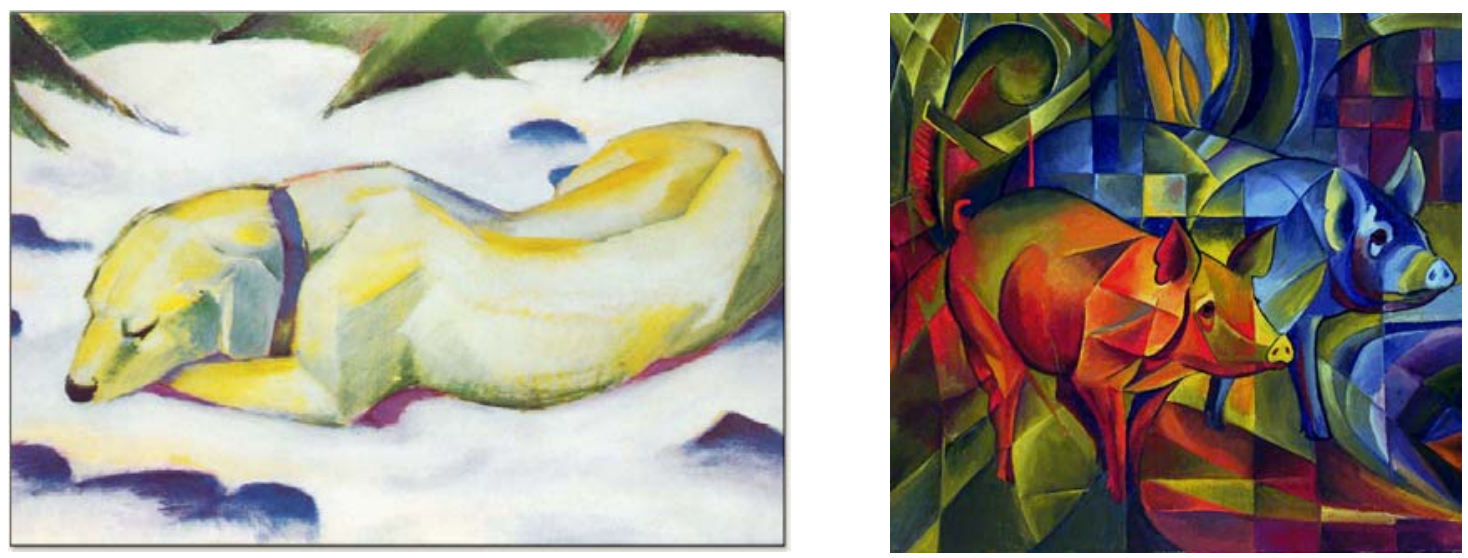

Figure S3. Two paintings by Franz Marc that illustrate various alternatives for the classical tonal shading technique. Here chromatic modulations in yellow-blue, and red-green are used. 


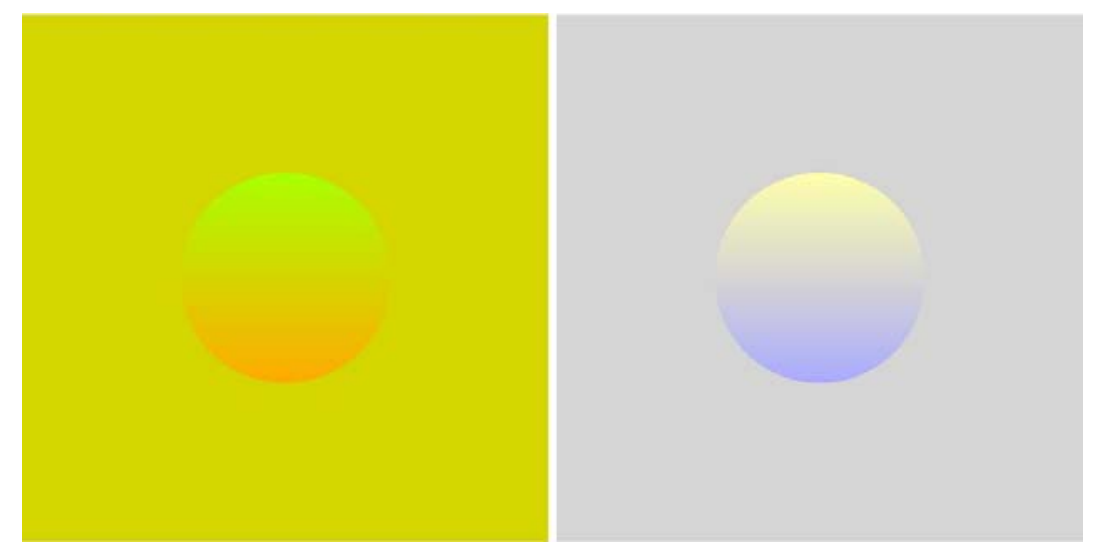

Figure S4. The case of proper shading emulated with chromatic modulation. This shows the conventional stimulus with tonal contrast replaced with chromatic contrast. We use a green-red modulation about yellow, and a yellow-blue modulation about gray. Hues are "balanced" in red:green:blue=1:1:1 ratios. In an "equi-luminant" rendering the green will be a factor of two darker than the red, the yellow a factor of seven darker than the blue. This means that the "yellow" will be dark brown, and so forth. "Equi-luminance" proper is simply not a reasonable option. "Gestalt balancing" is the way to go (see Figure S5).
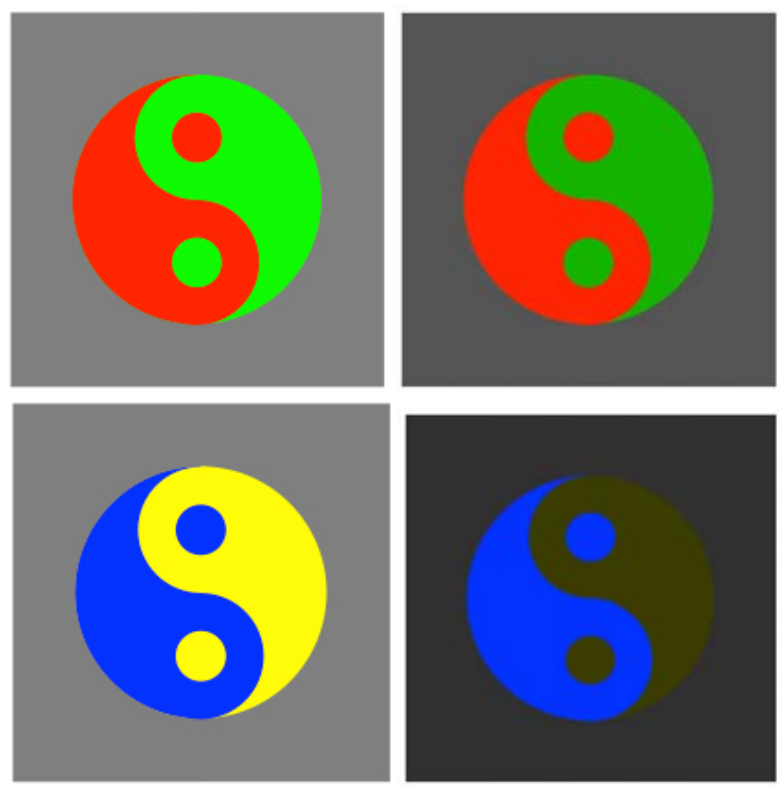

Figure S5. The Yang-Ying pattern in color. The left column uses red:green:blue=1:1:1, the right column red:green:blue=3:6:1. Notice that the blue in the equi-luminant (bottom right) condition is fully "out of balance" with the yellow-which even fails to look "yellow". 


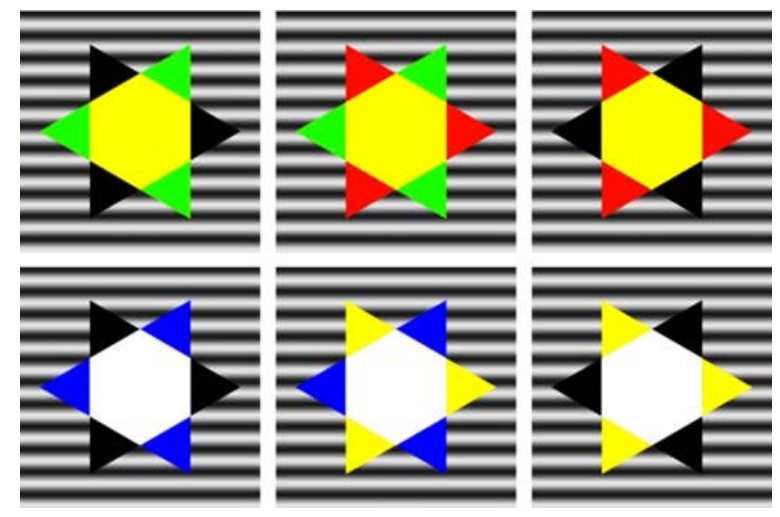

Figure S6. A "Gestalt grouping" balancing method. Depending upon the relative luminance of two color components, the center hexagon, which is the sum of the two component colors, tends to group with the color that is most like it. Notice that the background has no color or tone, it is indeterminate. The grouping occurs between the hexagon and a subset of the six triangles. Such experiments suggest that "Gestalt grouping power" is about equal for white-black-red-green-blue-cyan-purple-yellow, much as expected from the painter's perspective.
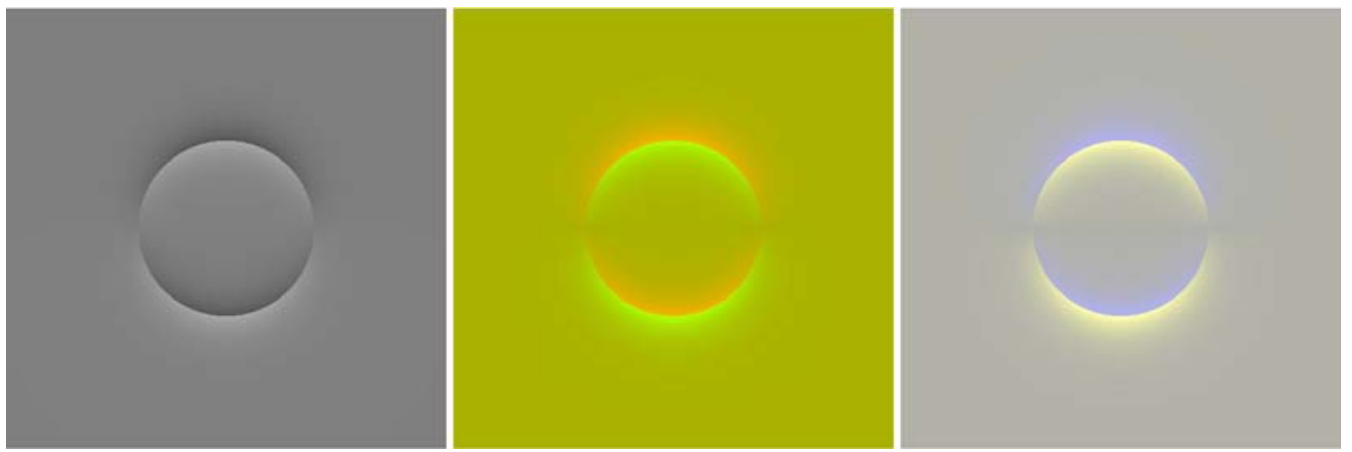

Figure S7. Examples of contour modulation by way of bipolar edges. In the image at far left the linear gradient inside the disk has been replaced with a uniform tone. The tonal area modulation ("shading proper") has been limited to a narrow strip about the circular outline. This clearly articulates pictorial relief. Substituting chromatic for tonal modulation yields the stimuli at center (green-red) and right (yellow-blue). 


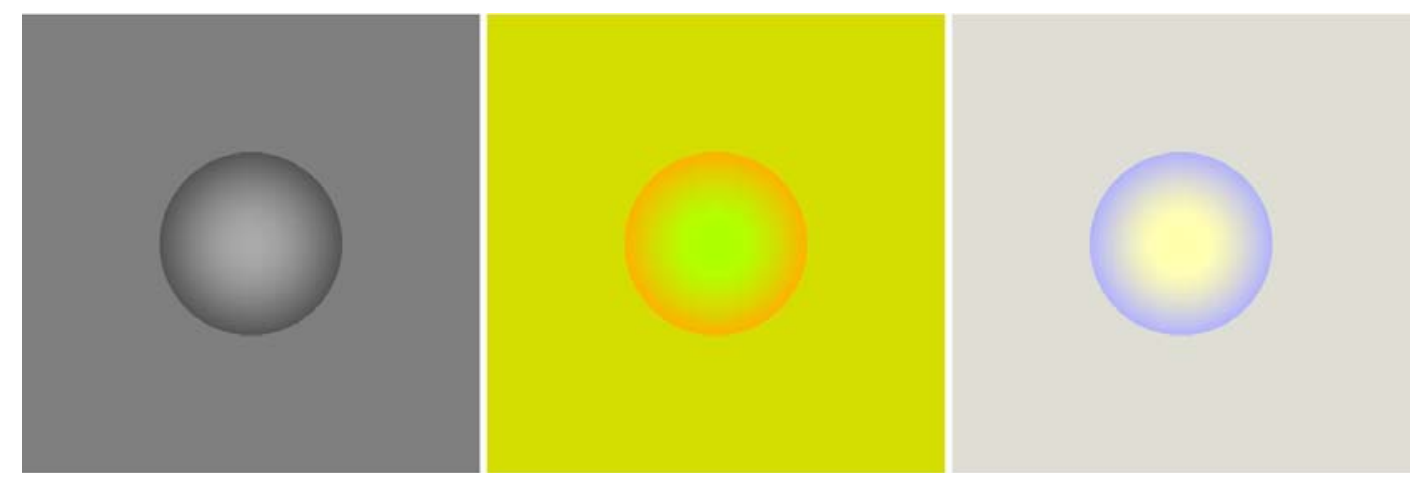

Figure S8. The case of edge darkening. In the image at far left the linear gradient inside the disk has been replaced with a tonal edge darkening. This sculptural shading - as it is often called - has no radiometric origin, yet it is quite effective. It is common in many cultures, and stylistic periods, because it does not introduce an irrelevant accident like a direction of illumination. At center and right chromatic modulation has been substituted for tonal modulation.
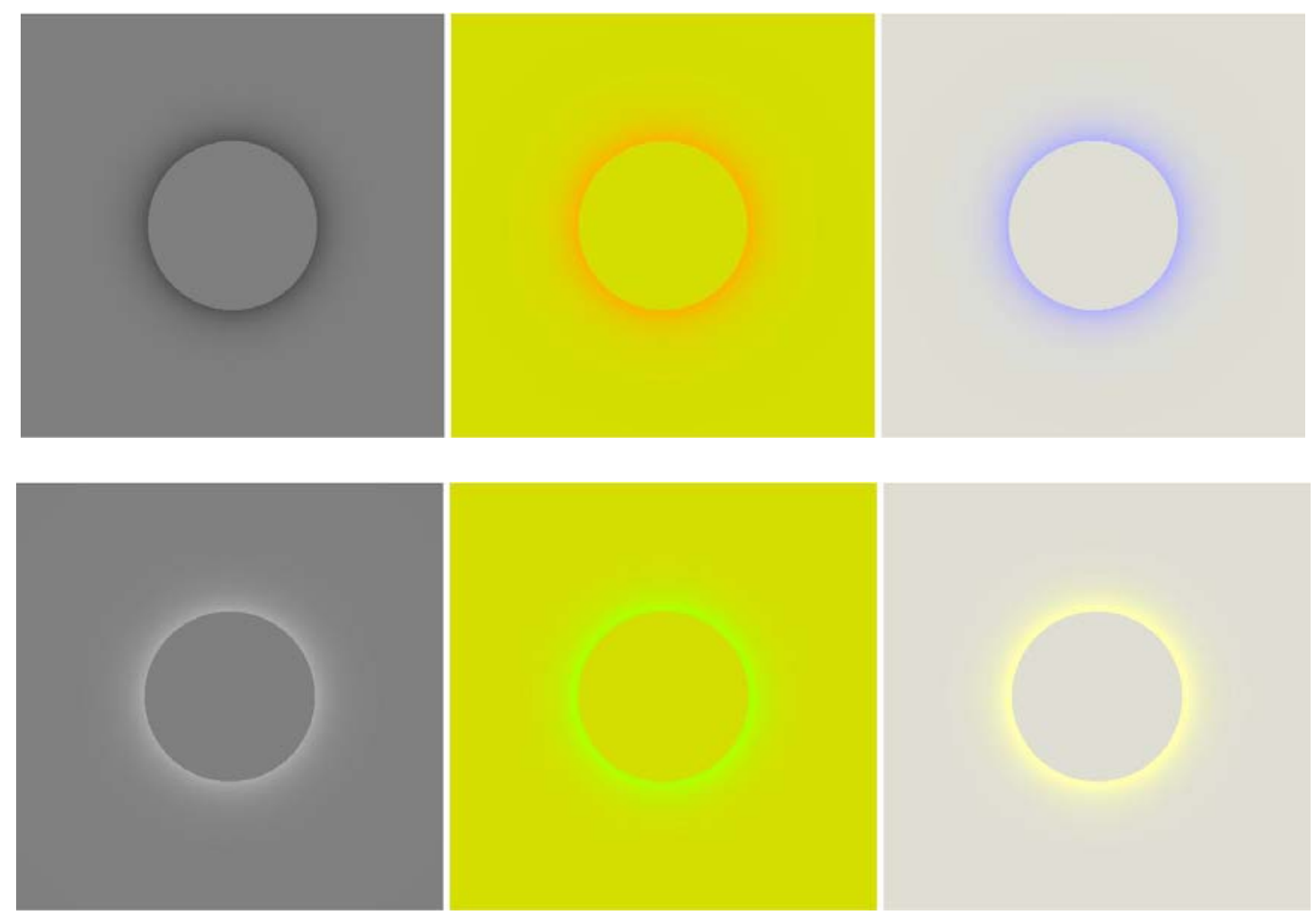

Figure S9. A number of stimuli, both tonal, and chromatic, that will almost certainly be perceived as "flat", although possibly raised or recessed from the picture plane. 


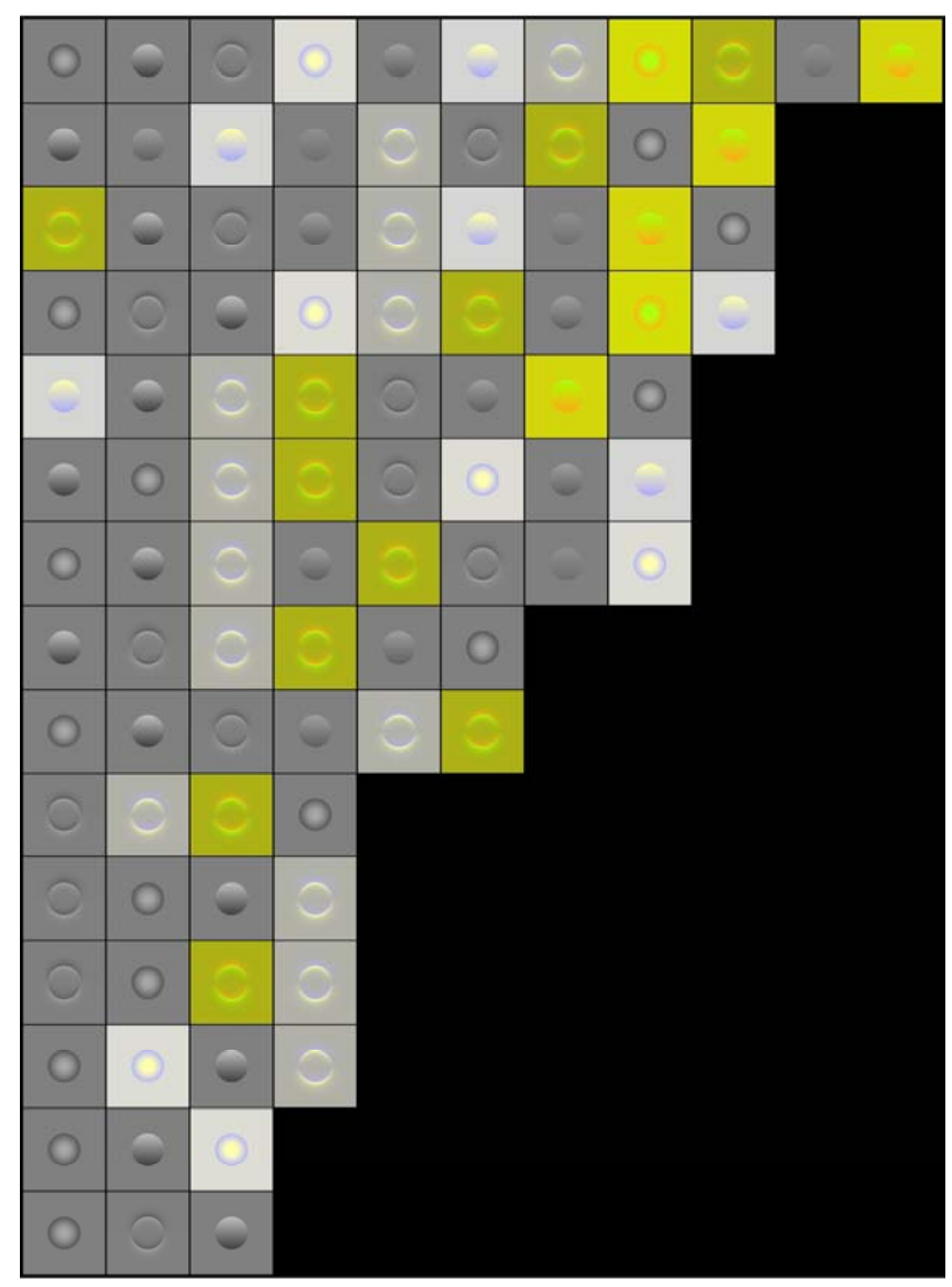

Figure S10. The initially sorted data. Judged articulation decreases from left to right, whereas the discriminative power of the observers decreases from top to bottom. 


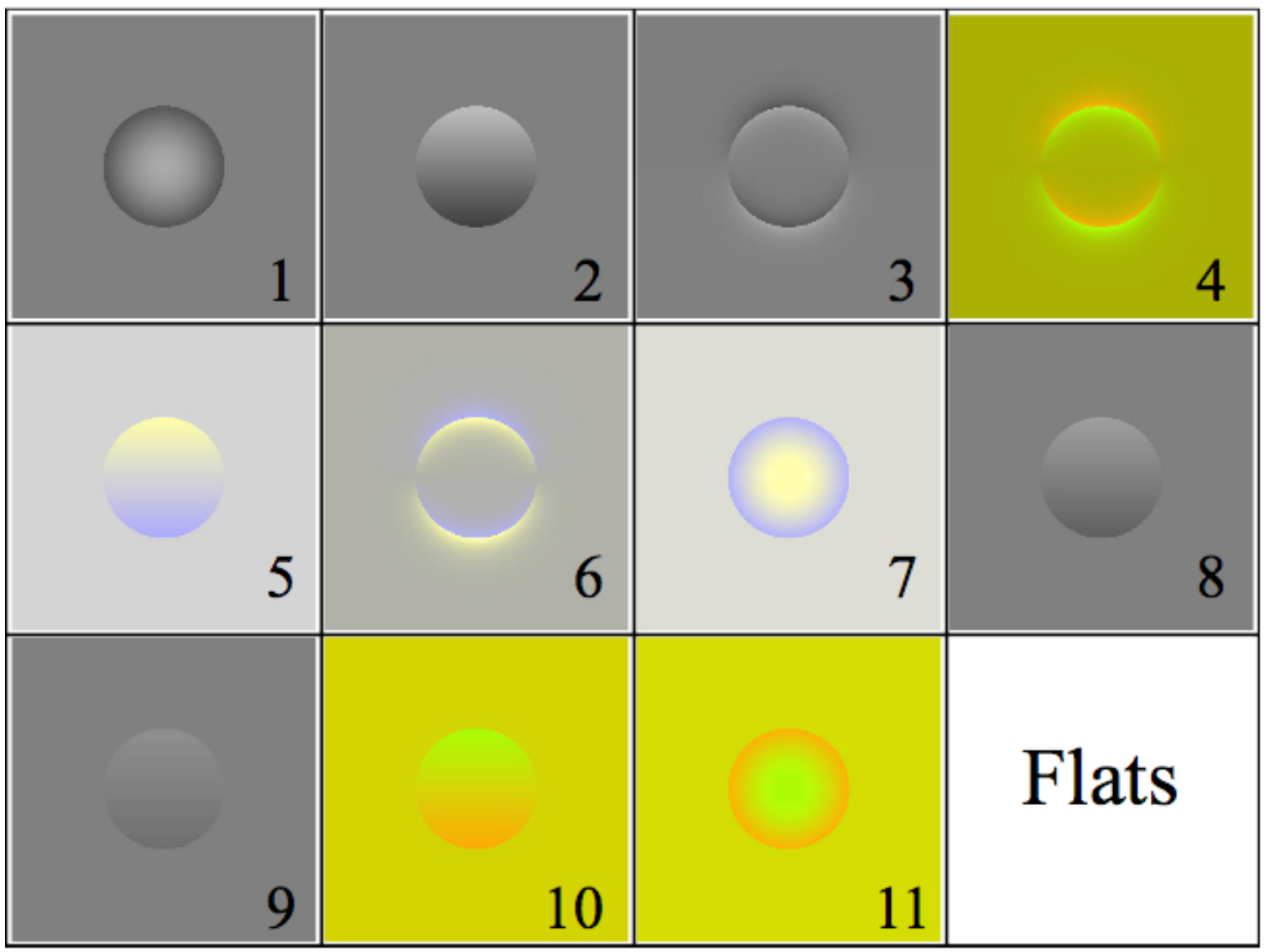

Figure S11. The overall order of articulation, to be read from left to right, top to bottom. Thus "edge darkening” is the overall winner. Several chromatically modulated patterns beat the lower contrast shaded instances. The order stops at rank twelve, where all observers confuse reliefs with flats. 